\title{
Escisión de voto en elecciones subnacionales en Colombia
}

\section{Split ticket voting in subnational elections in Colombia}

\section{Luz Helena Idrobo Bedoya (iD}

Universidad de Murcia, España

lh.idrobobedoya@um.es

\begin{abstract}
Resumen
La escisión del voto es un fenómeno electoral que se basa en elegir de forma diferenciada en dos elecciones celebradas en una misma jornada electoral. En este artículo se estudian las tres últimas elecciones a la alcaldía y el concejo en Bogotá, Cali, Medellín y Barranquilla, con el fin de hallar la magnitud del voto diferenciado en el ámbito subnacional. Para ello, se ha recurrido a un análisis con datos agregados con los cuales se ha podido aplicar el índice de escisión de Arian y Weiss. De esta manera, se ha logrado constatar empíricamente que en las elecciones locales, se presenta una escisión del voto en comicios concurrentes incentivada por factores tales como una participación diferente en cada elección concurrente, una alta fragmentación del sistema de partidos y una considerable volatilidad electoral.
\end{abstract}

Palabras clave: escisión de voto, elecciones subnacionales, participación electoral, comportamiento politico, fragmentación electoral.

\begin{abstract}
The split ticket voting is an electoral phenomenon that is based on choosing differently in two elections held on the same election day. This article examines the last three major and council elections in Bogota, Cali, Medellin and Barranquilla, in order to find the magnitude of the differentiated vote at the subnational level. To this end, an analysis has been used with aggregated data with which the Arian and Weiss spin-out index could be applied. In this way, it has been empirically noted that in local elections, there is a split ticket voting in concurrent elections incentivized by factors such as different participation in each concurrent election, high fragmentation of the party system and a considerable electoral volatility.
\end{abstract}

Keywords: Split ticket voting, subnational elections, electoral turnout, political behaviou, electoral fragmentation.

Articulo: Recibido el 18 de junio de 2020 y aprobado el 22 de octubre de 2020

\section{Introducción}

Las elecciones municipales o subnacionales han sido generalmente poco analizadas en el campo de la ciencia política (Delgado, 1999), debido en gran parte a la dificultad de estudiar un universo tan amplio de municipios en el que analizar unas elecciones nacionales resulta una mejor estrategia de investigación debido a la cantidad de información disponible (Riera et al., 2016). Sin embargo, se puede inferir que es relevante analizar el contexto subnacional debido a la ausencia de conocimiento profundo y la importancia que puede

\section{Cómo citar este artículo:}

Idrobo, LH. (2020). Escisión de voto en elecciones subnacionales en Colombia. Reflexión política 22(46), pp. 34-42. doi: https://doi.org/10.29375/01240781.3929 
tener en el entendimiento de dinámicas y tendencias del comportamiento electoral (Martín de la Cruz y García de Madariaga, 2019).

Así, en este artículo se propone un estudio de caso acotado a las cuatro grandes ciudades de Colombia, en el cual se analiza la escisión del voto en elecciones subnacionales con el propósito de generar un conocimiento lo más significativo posible del comportamiento electoral de las últimas tres elecciones (2011, 2015 y 2019) concurrentes a la alcaldía y el concejo en Colombia. Los comicios elegidos para su estudio han sido celebrados bajo unas reglas de juego que pretendían moderar el sistema de partidos y la lucha partidista (Giraldo y López, 2006; Muñoz et al., 2013).

De esta manera, el presente artículo pretende, mediante el análisis de la escisión de voto, realizar un aporte a los estudios de comportamiento electoral de elecciones subnacionales o de segundo orden ${ }^{1}$ en Colombia. Explica a su vez cómo la participación, la fragmentación y la volatilidad electoral son factores fundamentales de dicha escisión, puesto que gracias a la forma en que se desenvuelve el sistema electoral, es posible entender en qué magnitud ocurre el voto diferenciado en elecciones concurrentes.

\section{Conceptos clave}

El fenómeno de la escisión del voto ${ }^{2}$ es una conducta política basada en elegir de forma diferente entre dos comicios que confluyen en una misma jornada electoral: "Este fenómeno consiste así en un caso límite para las teorías del comportamiento electoral, ya que la convocatoria simultánea de elecciones supone un experimento natural en el que gran parte de las características sociodemográficas y actitudinales del elector permanecen constantes" (Sanz, 2008, p. 170). Los estudios sobre este tipo de conducta electoral se han centrado en la literatura norteamericana ${ }^{3}$, en la cual existen avances representativos que han permitido construir un marco conceptual en torno al fenómeno; sin embargo, sigue sin existir un acuerdo sobre cuál es la explicación más concreta para analizarlo (Riera, 2009). La literatura sobre el tema se clasifica según múltiples cuestiones, evaluando casos de todo tipo en el ámbito democrático.

En este sentido, "la escisión del voto, con su correlato del voto diferencial, ha recibido diversas denominaciones, algunas de ellas más descriptivas que técnicas” (Montero, 1988, p. 182). Algunas explicaciones se han decantado por dos tipologias diferentes. En primer lugar, ciertos autores se han enfocado en las características individuales y la lealtad de partido como explicación de este fenómeno. Por otro lado, hay estudios sobre este fenómeno que se basan en la explicación sobre la preponderancia de los candidatos y sobre todo la visibilidad que tienen como políticos experimentados (Beck et al., 1992). Otras concepciones se centran en si se aplica a elecciones de tipo concurrente o no. Puesto que "Cuando las elecciones son concurrentes, las campañas de los partidos se superponen y la información disponible se centra en un conjunto de temas más pequeño" (Colomer, 2001, p. 181). Mientras que si las elecciones no son concurrentes hay más posibilidad, como afirma Colomer, de que exista un voto dividido estratégico.

Esta concepción del voto dividido estratégico privilegia al votante sofisticado. Su propósito es entender los efectos que tiene la conducta electoral dividida del votante en la configuración de los gobiernos. Aunque es una concepción interesante, solo tiene en cuenta al elector como un agente racional, donde la explicación pasa por analizar los incentivos que tienen los votantes para dividir su voto entre dos o más opciones. Sin embargo, este tipo de explicaciones componen más los análisis del gobierno dividido que la explicación del voto. Además, este tipo de análisis son mucho más complicados de explicar a nivel empírico, ya que obtener datos confiables dentro de los cuales se pueda evidenciar una estrategia del elector para privilegiar la configuración de gobierno resulta bastante complejo, puesto que, al no participar en elecciones concurrentes, las variables cambian

${ }^{1}$ El concepto de elecciones de segundo orden parte de las investigaciones en la década de los ochenta de Reif y Schmitt (1980), quienes analizan las elecciones en un contexto europeo. En su análisis plantean que las elecciones europeas son elecciones de segundo orden que responden a intereses distintos a los de las elecciones nacionales o territoriales de cada país. Por tanto, con esta acepción se hace una apuesta por analizar las distintas arenas en las que se presentan las elecciones, como una forma de analizar las elecciones y evidenciar el comportamiento electoral del votante.

${ }^{2}$ Se opta por examinar la escisión de voto, ya que en español es el término que se usa para explicar el split ticket voting. Esta limitación se realiza por el carácter simultáneo de las elecciones en las que se divide el voto, ya que existen otras variaciones relacionadas, pero que no cumplen con el criterio de caracter simultaneo de las elecciones en las gue se divide
simultaneidad de comicios (Trujillo et al., 2015, pp. 34-35).

${ }^{3}$ Especialmente en los Estados Unidos, en el que se acuña el fenómeno como split ticket voting: "un boleto se divide si el votante i vota por el partido j en

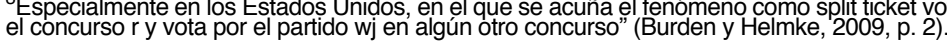


de una elección a otra, imposibilitando un análisis concreto. Asimismo, el perfil de votante sofisticado también implicaría que las percepciones de los votantes sean estratégicas y tengan la necesidad de percibir qué partidos tienen mayores posibilidades, o si su partido es el más adecuado. Ante estas premisas se corre el riesgo de ser bastante ambiguos y las explicaciones dependerían de la sinceridad del votante a la hora de realizar una encuesta o entrevista (Colomer, 2001).

En esta misma línea de análisis, se encuentra el llamado voto dual (Pallarés et al., 2000; Riba, 2000; Riba y Boix, 2000), como un fenómeno que responde a la transferencia de votos entre partidos según el tipo de elecciones. De tal manera, este tipo de voto cuenta con dos dimensiones. En primer lugar, los traspasos de votos entre elecciones y partidos, evidenciando una suerte de detrimento electoral. Por otra parte, está la segunda dimensión, la cual se refiere a los logros electorales, es decir, los votos que se han conseguido en una elección y que no tenían con anterioridad (Montero y Font, 1991). En este sentido, el voto dual fue instituido a partir del análisis de las elecciones autonómicas y generales en Cataluña, separadas en el tiempo la una de la otra. Al ser no concurrentes sus valoraciones se centran en la ideología y en el equilibrio que esperan tener los votantes con un voto diferente en cada tipo de elección.

De esta manera, en el caso del voto dual,

[...] el principal argumento parece ser el de que estos votantes están convencidos de que cada una de las dos fuerzas políticas es la que mejor defiende sus intereses en el ámbito de sus respectivas competencias. En general, son votantes de ideología centrista que quieren estabilidad y gobiernos fuertes y eficaces (Riba, 2000, p. 62).

Esta concepción, por tanto, aboga por una intencionalidad de mantener un equilibrio de poder entre los gobiernos. Sin embargo, al no ser concurrentes, las variables sociodemográficas no son constantes y los votantes pueden ser diferentes entre elecciones perjudicando las explicaciones finales. Además, incluyen la variable nacionalista en el seno de un Estado, con lo cual para nuestro análisis no resultaría útil, ni pertinente.

Por otro lado, donde mejor se puede analizar la división del voto es un contexto electoral en el que el elector percibe de la misma manera las elecciones del mismo nivel (Roscoe, 2008), como por ejemplo las elecciones locales concurrentes, donde los mismos electores se ven expuestos a dos decisiones al mismo tiempo. Así, el fenómeno de la escisión del voto se refiere a un comportamiento individual en el que se vota en una misma jornada de forma diferente a dos partidos. Su estudio es alimentado principalmente de encuestas y datos agregados (Gitelson y Bayer, 1983).

\subsection{Modelos clásicos de estudio del análisis del Split ticket voting}

Después de realizar un análisis conceptual de las diferentes concepciones y factores alrededor de la escisión del voto, resulta relevante señalar los modelos teóricos más importantes en torno a este fenómeno electoral. El primer enfoque defiende un modelo de compensación o intencionado promovido por Fiorina (1992), en el que hay una explicación sobre el concepto de la ideología donde la compensación se establece en la diferencia que tiene el votante entre dos opciones políticas.

El segundo enfoque hace referencia al papel que tienen los partidos políticos y las ofertas que presentan dichas agrupaciones en una elección $\mathrm{u}$ otra según Jacobson (1991), además de evidenciar la relevancia de la oferta política en cada uno de los comicios. En un último enfoque clásico, las investigaciones se han centrado en entender la escisión del voto como un acto de voto estratégico (Karp et al., 2002; Karp y Garland, 2007). En este último enfoque se pueden encontrar también dos posibles causas para dividir el voto en una misma jornada electoral en dos comicios. La primera causa aboga por las diferencias en el sistema electoral y la segunda causa se centra en las diferencias entre lo que se encuentra en juego para cada convocatoria electoral (Sanz, 2007).

\section{2. Índice de escisión del voto de Arian y Weiss}

En otro orden de ideas, una técnica relevante para el análisis de la escisión del voto es la aplicada en Israel $^{4}$ por Arian y Weiss. Sus aportes han generado un índice que "pondrá de manifiesto la medida en

${ }^{4}$ Política y administrativamente, el gobierno local en Ișrael depende de los partidos políticos y los ministerios del gobierno que operan desde el centro. La política israelí, por razones históricas y estructurales, siempre ha estado dominada por el poder nacional, en lugar del local. Desde antes del establecimiento del Estado en 1948, una ideologia agraria, casi anti urbana, ha dominado los partidos politicos importantes del pais. El tamano relativamente pequeno del pais y la novedad de los colonos tendian a impedir el establecimiento de bases regionales de division. Esta falta de autonomia local coincidio con nacionales como la Agencia Judía y la Histadrut y, después de la independencia, por el propio gobierno (Arian y Weiss, 1969, p. 375). 
la que los ciudadanos de un ámbito dado votan por partidos distintos en diferentes tipos de elecciones" (Ocaña y Oñate, 1999, p. 241). El índice se representa en:

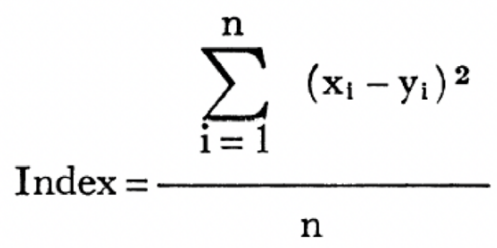

Si tenemos en cuenta el análisis de Montero, se observa que "la $\mathrm{x}$ es el porcentaje de votos de cada partido en las elecciones autonómicas; y, el porcentaje de votos de cada partido en las elecciones legislativas, y, n, el número de partidos considerado" (1988, p. 185). Por tanto, este índice merece toda la atención al analizar elecciones en distintos niveles y al hacer comparaciones entre elecciones. Así, los autores del índice sostienen que, a partir de las estadísticas electorales, se podría observar cómo los votantes votan por partidos distintos a nivel nacional, probablemente con el fin de recompensar algún proyecto político que haya cumplido su labor.

Aunque adaptado al contexto israelí, el indice ha sido probado en otros casos, como por ejemplo, en el español, donde se logró establecer las diferencias entre las elecciones autonómicas y generales en el año 1986 en la comunidad andaluza, "dado que permite tener en consideración tanto los votos diferenciales obtenidos por cada partido, como el número total de los que han tenido un cierto protagonismo en el intercambio de votos" (Montero, 1988, p. 185). Así, este índice permitirá observar el nivel de escisión de voto en las principales ciudades de Colombia en las elecciones a la alcaldía (ejecutivo local) y el concejo municipal (corporación administrativa local).

\section{Elecciones subnacionales en Colombia (2011-2019)}

En este epígrafe se procede a estudiar las elecciones subnacionales elegidas. En primer lugar, se observa cómo ha sido la participación electoral de los ciudadanos de las cuatro ciudades seleccionadas. Se realizó una descripción de los datos electorales y de esta forma, se evidenciaron las diferencias entre los comicios municipales a la alcaldía y el concejo municipal (Losada, 2012, p. 193); diferencias que son fundamentales para la aplicación del índice de escisión de Arian y Weiss. Se hizo un aporte hacia la comprensión de los estudios subnacionales mediante un análisis multidimensional (Fleischmann y Stein, 2017).

Así, en una comparativa de las tres elecciones a la alcaldía se puede observar que la participación electoral se ha incrementado de un evento a otro en la mayoría de las ciudades. Se exceptúa la ciudad de Medellín, donde la participación ha sido mayor en el 2011 que en 2015, pero mayor en el 2019. Por otra parte, Barranquilla ha presentado un comportamiento de mayor participación en las elecciones del 2015 y menor en el 2019. De tal forma, los datos evidencian que, aunque se ha elevado en algunos momentos y ciudades la participación, esta sigue siendo baja en este tipo de comicios (Rodríguez y García, 2016).

Tabla 1. Participación en elecciones a la alcaldía y el concejo de 2011, 2015 y 2019

\begin{tabular}{ccccccccc}
\multirow{2}{*}{ Elecciones } & \multicolumn{2}{c}{ Bogotá } & \multicolumn{2}{c}{ Cali } & \multicolumn{2}{c}{ Medellín } & \multicolumn{2}{c}{ Barranquilla } \\
\cline { 2 - 10 } & Alcaldía & Concejo & Alcaldía & Concejo & Alcaldía & Concejo & Alcaldía & Concejo \\
\hline 2011 & 47,41 & 45,82 & 42,84 & 42,39 & 50,69 & 49,03 & 47,44 & 46,91 \\
\hline 2015 & 51,5 & 49,8 & 45,4 & 44,8 & 49,6 & 48,4 & 54,7 & 54,3 \\
\hline 2019 & 55,22 & 53,9 & 48,6 & 47,9 & 51,3 & 50,6 & 53,2 & 52,9
\end{tabular}

Fuente: Elaboración propia con datos de la Registraduría Nacional del Estado Civil de Colombia.

En el caso de la alcaldía, los resultados exponen una proliferación de plataformas de corte personalista que han desplazado a los partidos tradicionales en la mayoría de las ciudades a excepción de la ciudad de Barranquilla, donde todavia se observa un apoyo a los partidos consolidados. Asimismo, estos partidos nuevos han logrado concentrar el voto (Mainwaring et al., 2010) de la alcaldía estableciendo una tendencia a la prevalencia de grupos significativos o plataformas electorales en las elecciones a la alcaldía posteriores a las reformas electorales de 2003, 2009 y 2011. 
Por su parte, las elecciones al concejo municipal también evidencian un incremento de la participación en el año 2019 en casi todas las ciudades. Barranquilla es el único caso en el cual ha sido más bajo que en el evento electoral anterior. Asimismo, la ciudad de Medellín tuvo un pequeño descenso en la participación en el año 2015 en comparación con los registrados en el año 2011. De esta manera, la participación se mantuvo al alza entre eventos electorales pero en el concejo se evidencia aún más abstención que en las elecciones a la alcaldía. En el mismo sentido, se ha podido constatar que los votos en blanco para esta elección fueron una constante para esta corporación administrativa con unos porcentajes significativos.

Así las cosas, las elecciones al concejo municipal han demostrado tener aún una influencia significativa de los partidos tradicionales o consolidados (más de 10 años en la lucha electoral) (Mainwaring et al., 2017), en la elección de esta corporación. Se manifiesta con ello que a pesar de la alta fragmentación, estos partidos tienen su nicho electoral en este tipo de elecciones, por lo menos en las cuatro ciudades analizadas. Asimismo, se ha evidenciado que la ciudad que mantiene un mayor reducto electoral de los partidos consolidados es Barranquilla, mientras que el caso contrario es Medellín, donde existe una presencia continuada de fuerzas políticas creadas por movimientos ciudadanos o recogidas de firmas. Por su parte, Cali y Bogotá, aunque también tienen presencia de los partidos tradicionales para el concejo, estos tienen que compartir su lugar de mayoría con otros partidos ya consolidados como el partido de la U en Cali o la Alianza Verde y el Polo Democrático en el caso de la capital.

Tabla 2. Fraccionalización en las elecciones a la alcaldía y el concejo 2011, 2015 y 2019

\begin{tabular}{ccccccccccc}
\multicolumn{1}{c}{} & & NEPe & \multicolumn{3}{c}{ Hiperfraccionalización } & \multicolumn{2}{c}{ NP } \\
\hline \multirow{2}{*}{ Alcaldía } & 2011 & 2015 & 2019 & 2011 & 2015 & 2019 & 2011 & 2015 & 2019 \\
\hline & Bogotá & 5 & 4 & 4 & 5,2 & 4,3 & 1,7 & 3,5 & 3,2 & 3 \\
\hline & Cali & 4 & 4 & 4 & 4,4 & 4,4 & 1,7 & 2,17 & 3 & 4 \\
\hline \multirow{2}{*}{ Concejo } & Medellín & 3 & 4 & 4 & 3,2 & 1,7 & 1,6 & 3 & 3 & 3 \\
\hline & Barranquilla & 2 & 2 & 2 & 2,4 & 1,2 & 1,4 & 1,4 & 1 & 1 \\
\hline & Bogotá & 11 & 15 & 13 & 7,1 & 7,6 & 2,15 & 10 & 12 & 7 \\
\hline & Cali & 10 & 11 & 12 & 10,02 & 6,05 & 2,47 & 9 & 10 & 10 \\
\hline & Medellín & 9 & 12 & 12 & 6,8 & 6,17 & 2,18 & 7 & 9 & 5 \\
\hline
\end{tabular}

Fuente: Elaboración propia con datos de la Registraduría Nacional del Estado Civil de Colombia.

En cuanto al análisis de la fragmentación del sistema de partidos, se han calculado tres índices relevantes en un tipo de sistema como el colombiano para medir la concentración del poder político en las elecciones locales. Sin embargo, la comparativa evidencia un nivel medio alto de fragmentación en las elecciones a la alcaldía, mientras que en las elecciones al concejo se presenta un nivel alto de fragmentación de partidos que impide hablar de mayorias en una corporación que tiene como objetivo ejercer un control y apoyar los proyectos del ejecutivo local, a pesar de que el concejo no tiene una función determinante como en el sistema de partidos subnacional español. Esta fragmentación puede provocar el retraso $\mathrm{u}$ obstrucción de proyectos prioritarios en las grandes ciudades analizadas.

Por otro lado, a pesar de que el índice de hiperfraccionalización (I) esté sujeto a controversia, dada su sensibilidad a los partidos muy pequeños, su cálculo permite esbozar el panorama altamente fragmentado en un contexto subnacional (Ruiz y Otero, 2013). De esta manera, se ha recurrido al índice Molinar NP con el fin de contrarrestar dichas restricciones del I. Así, el NP ha evidenciado la competitividad del sistema, dándole un valor importante en el cálculo al partido mayoritario. De esta manera, el NP puede ser el más indicado para entender cómo es el sistema de partidos subnacional de estas ciudades colombianas. 
Igualmente, el NP ha permitido observar un sistema de partidos más cercano a los resultados electorales, donde se evidencia en el caso de la alcaldía una lucha entre máximo tres fuerzas en las ciudades de Bogotá, Cali y Medellín. Mientras que en la ciudad de Medellín los valores del NP han mostrado la prevalencia de un partido mayoritario en las tres elecciones analizadas. Por su parte, el NP del concejo municipal, refleja al igual que los otros índices una alta fragmentación de partidos.
Sin embargo, ha evidenciado una tendencia a su moderación desde las elecciones del 2011 a las últimas celebradas en el año 2019. Así, se comprueba que el NP podría reflejar más fielmente el sistema de partidos subnacional de Colombia, dado que en las elecciones a la alcaldía el sistema de elección es por mayoría simple, en el cual un partido se lleva todo, mientras que en el concejo municipal los datos de NP evidencian la lucha de los partidos que tienen una significación electoral.

Tabla 3. Volatilidad en las elecciones a la alcaldía y el concejo (2011-2019)

\begin{tabular}{ccccc} 
& Bogotá & Cali & Medellin & Barranquilla \\
\hline Volatilidad electoral total $^{\mathbf{5}}$ & & & & \\
\hline $2011-2015$ & 6,7 & 18,5 & 26,2 & 10,3 \\
\hline $2015-2019$ & 16,5 & 14,8 & 11,1 & 14,2 \\
\hline Volatilidad extra sistémica & & & & \\
\hline $2011-2015$ & 3,6 & 8,4 & 22,9 & 5,6 \\
\hline $2015-2019$ & 8,5 & 7 & 10 & 4,7
\end{tabular}

Fuente: Elaboración propia con datos de la Registraduría Nacional del Estado Civil de Colombia.

En cuanto al análisis de la volatilidad, se ha detectado mediante el índice total electoral que la volatilidad fue mayor en Cali y Medellín entre las elecciones de 2011 a 2015 que entre 2015 a 2019. Sin embargo, en Bogotá y Barranquilla la volatilidad fue mayor entre 2015 a 2019. Esta situación de lealtad de los votantes consigue generar outsiders políticos que pueden acceder mediante plataformas personalistas al poder, evidenciando que las ideologías o etiquetas de partidos son más débiles y existe una gran posibilidad de abrirle paso a nuevos partidos entre elecciones (Mainwaring y Zoco, 2007, p. 150).

Sin embargo, se puede decir que la volatilidad extra sistémica puede dar mayores pistas sobre el sistema de partidos subnacional que se está analizando. De esta forma, si se siguen los planteamientos de Mainwaring, Gervasoni y España-Nájera sobre la importancia de calcular la volatilidad extra sistémica, se puede decir que este índice permite observar de manera más clara cómo es el sistema de partidos en un contexto donde entran y salen múltiples fuerzas políticas entre eventos electorales. Este hecho se creería que podría impedir un fortalecimiento o afianzamiento del sistema de partidos (Luján y Schmidt, 2018) (tal y como lo tenía previsto las distintas reformas electorales), además de generar una mayor tendencia a la escisión del voto. Así, al obtener los valores de la volatilidad extra sistémica se puede decir que la ciudad con una menor influencia de las nuevas fuerzas electorales es Barranquilla. Mientras que se evidencia una mayor incidencia de esta volatilidad en ciudades como Medellín, Cali y por último, Bogotá, debido probablemente a la alta fragmentación en las elecciones municipales de 2015 (Rodríguez y García, 2016).

\subsection{La escisión del voto en las elecciones locales en Colombia (2011-2019)}

En cuanto a la manera como se ha analizado la escisión del voto en distintos contextos y países, esta investigación elige seguir la estela de los análisis de dirección horizontal en los que se analizan elecciones de un mismo nivel (Sanz, 2008). De esta forma, se opta por realizar un análisis entre elecciones que

\footnotetext{
${ }^{5}$ El índice de volatilidad electoral permite entender "el trasvase agregado de votos de un partido a los otros entre distintas elecciones" (Mainwaring y Torcal, 2005, p. 147), reflejando los cambios en las opciones de los votantes, además de los cambios internos partidistas (fusiones o divisiones) (Mainwaring y

Í́ndice de volatilidad extra sistémica de Mainwaring, Gervasoni y España-Nájera que indica ciertas disconformidades de los electores con los partidos establecidos o en este caso tradicionales, mostrando así su insatisfacción y su distanciamiento con ellos. Con lo cual se puede observar que las lealtades pueden cambiar en cuanto hay nuevos partidos en cada lucha electoral que logran atraer el voto (2010, p. 32).
} 
están en un mismo nivel de gobierno (local), para estudiar la magnitud en un ámbito intra-nivel (Burden y Helmke, 2009) donde se exploran elecciones a un cargo ejecutivo por elección mayoritaria y cargos a corporaciones administrativas como el concejo municipal elegidos bajo un sistema proporcional.

Igualmente, al remitirnos a la forma de abordar el análisis de este fenómeno, se optará por analizar la magnitud del voto diferenciado desde los datos resultantes de la aplicación del índice de escisión del voto (Arian y Weiss, 1969), el cual permitirá adaptar un índice relevante al caso colombiano con elecciones concurrentes de dirección horizontal. Se incentiva con ello la posibilidad de cuantificar la magnitud de esta escisión en las cuatro ciudades analizadas en las elecciones locales a la alcaldía y el concejo municipal de los años 2011, 2015 y 2019.

Así, al analizar con datos agregados la escisión del voto (Arian y Weiss, 1969; Burden y Kimball, 1998; Riera et al., 2016), se ha podido constatar a través de la aplicación del índice de escisión de voto (IEV) que en las cuatro ciudades analizadas no se presenta un comportamiento electoral homogéneo en elecciones concurrentes y existe un elevado nivel de voto diferenciado en las elecciones al alcalde y el concejo municipal. Asimismo, se ha retratado que la participación hacia la alcaldía es mayor que la que se da para el concejo municipal en las tres últimas elecciones.

Gráfico 1. Comparativa del IEV de las elecciones a la alcaldia y el concejo (2011-2019)

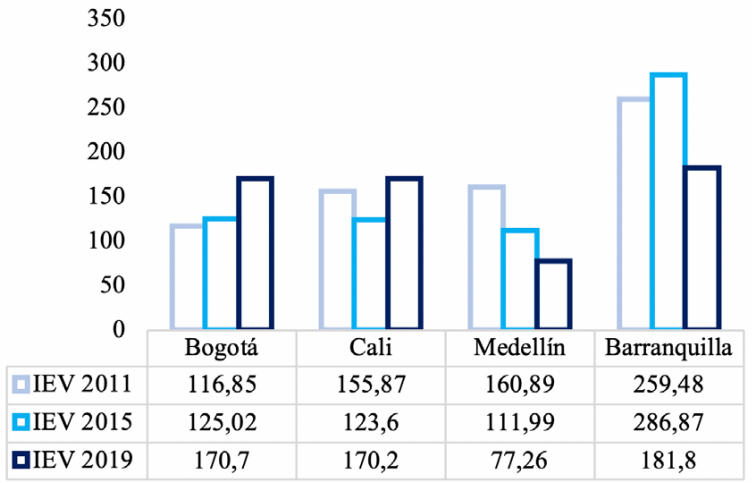

Fuente: elaboración propia con datos agregados electorales Resultados electorales municipales 2011-2019.

Si bien los datos permiten corroborar la hipótesis de Arian y Weiss sobre el incremento de la escisión de voto entre eventos electorales, en los casos analizados se ha observado que solo en las ciudades de Bogotá y Cali se ha presentado el incremento de la escisión, mientras que en Medellín y Barranquilla decreció en la última cita electoral municipal en Colombia. Estas tendencias reflejan a grandes rasgos aspectos relevantes de unas elecciones locales (Delgado, 2010), como por ejemplo, el declive de los partidos tradicionales colombianos y la presencia de plataformas independientes nuevas en cada una de las elecciones analizadas.

De igual manera, los datos de participación han corroborado que en las grandes ciudades de Colombia existe un elevado abstencionismo (Ángulo, 2016) que se traduce en unas bajas cuotas de participación en casi todos los comicios celebrados en el país. Asimismo, al analizar los datos de participación y diferencias entre comicios concurrentes se ha podido constatar que no solo el voto diferenciado existe, sino que este fenómeno es un proceso de reflexión compleja del electorado, donde la cultura del país en cuanto a la participación electoral puede despejar algunos de las interrogantes sobre el comportamiento diferenciado de los votantes colombianos (Montabes, 1996).

\section{Conclusiones}

En términos generales estas líneas han pretendido evidenciar un fenómeno electoral que normalmente se estudia a nivel nacional. De esta forma, los hallazgos aquí consignados significan que la escisión del voto en elecciones concurrentes es una tendencia en las grandes ciudades de Colombia y es un comportamiento que se encuentra influido por la gran fragmentación, la alta volatilidad del sistema de partidos subnacional colombiano y la baja participación electoral.

Asimismo, se advierte que al ser un primer acercamiento al análisis empírico de la escisión del voto en un ámbito subnacional, se ha establecido cuál es la magnitud de este fenómeno en comicios concurrentes locales, donde se ha podido observar que este fenómeno está presente en las cuatro ciudades analizadas y su valor elevado se produce probablemente por razones accidentales, dada la alta personalización de la política y la distinción e importancia que otorgan los ciudadanos a las dos elecciones locales (Jacobson, 1991).

De las elecciones subnacionales, se ha podido constatar que el IEV es alto en la mayoría de las ciudades debido a la alta fragmentación del sistema de partidos en las elecciones al 
concejo municipal y al gran trasvase de votos entre elecciones concurrentes. Esta situación presenta un panorama bastante complejo, donde no se presenta un straight vote en los términos de Campbell y Miller (1957), sino que hay una decisión de gran parte del electorado de elegir de forma distinta en las elecciones a la alcaldía y el concejo que se celebran de forma simultánea. Lo cual plantea que esta situación puede suceder, dada la baja identificación partidista por parte del votante, que se afianza con la significativa aparición de partidos y fuerzas políticas nuevas en cada elección analizada. Igualmente, se puede decir que podrían existir razones accidentales (Jacobson, 1991), dada la alta personalización de la política y la distinción e importancia que otorgan los ciudadanos a las dos elecciones locales. Sin embargo, estas hipótesis derivadas del enfoque accidental tendrían que sustentarse con datos de encuestas.

En definitiva, los datos de este artículo describen la magnitud del fenómeno de la escisión del voto. El siguiente paso es identificar los factores que influyen en este comportamiento, teniendo en cuenta las características de cada territorio y las dinámicas políticas que se presentan en torno a lucha electoral en elecciones subnacionales. De tal manera, este artículo propone seguir avanzando en el análisis del comportamiento diferenciado de los electorales colombianos en las elecciones subnacionales. Así, se subraya la necesidad de tener encuestas lo bastante robustas y significativas de cada ciudad que permitan generar unos modelos de voto que ayuden a entender cuáles son los factores determinantes que incentivan esa escisión del voto en estas elecciones concurrentes.

\section{Referencias}

Ángulo, M. (2016). Intención de voto y simpatía partidista en Bogotá. Colombia Internacional, 86(Enero-abril), 81-106. http://dx.doi.org/10.7440/colombiaint86.2016.03

Arian, A., \& Weiss, S. (1969). Split-Ticket Voting in Israel. The Western Political Quarterly, 22(2), 375-389.

Beck, P. A., Baum, L., Clausen, A. R., \& Smith, C. E. (1992). Patterns and Sources of Ticket Splitting in Subpresidential Voting. The American Political Science Review, 86(4), 916-928.

Burden, B. C., \& Helmke, G. (2009). The comparative study of split-ticket voting. Electoral Studies, 28, 1-7. https://doi.org/10.1016/j.electstud.2008.06.004

Burden, B. C., \& Kimball, D. C. (1998). A New Approach to the Study of Ticket Splitting. The American Political Science Review, 92(3), 533-544.
Campbell, A., \& Miller, W. E. (1957). The motivational basis or straight and split ticket voting. The American Political Science Review, 51(2), 293-312.

Colomer, J. M. (2001). Instituciones politicas. Ariel.

Delgado, I. (1999). Resultados electorales y orientación del voto en los comicios municipales de 1995. Revista española de investigaciones sociológicas Reis, 86, 247-273.

Delgado, I. (2010). Elecciones municipales en España. dimensiones analíticas y aspectos distintivos de ocho procesos electorales (1979-2007). Política y sociedad, 47(3), 13-36.

España-Nájera, A., y Martínez, M. (2020). Cómo medir la congruencia: comparando tres medidas en América Central. Reis. Revista Española de Investigaciones Sociológicas, 169(1), 63-84.

Fleischmann, A., \& Stein, L. (2017). Campaign Contributions in Local Elections. Political Research Quarterly, 51(3), 673-689. https://www.jstor.org/stable/3088044

Giraldo, F., y López, J. D. (2006). El comportamiento electoral y de partidos en los comicios para Cámara de representantes de 2002 y 2006: Un estudio comparado desde la reforma política. Colombia Internacional, 64(jul-dic), 122-153.

Gitelson, A., \& Bayer, P. (1983). Ticket-Splitting : Aggregate Measures vs . Actual Ballots. The Western Political Quarterly, 36(3), 410-419.

Jacobson, G. (1991). Explaining Divided Government: Why Can't the Republicans Win the House? PS: Political Science and Politics, 24(4), 640-643. https://doi.org/10.2307/419393

Jacobson, G. C. (1991). Explaining Divided Government: Why Can ' t the Republicans Win the House? PS: Political Science and Politics, 24(4), 640-643.

Karp, J. A., \& Garland, M. W. (2007). Ideological ambiguity and split ticket voting. Political Research Guarterly, 60(4), 722-732. https://doi. org/10.1177/1065912907306761

Karp, J. A., Vowles, J., Banducci, S. A., \& Donovan, T. (2002). Strategic voting, party activity, and candidate effects: testing explanations for split voting in New Zealand's new mixed system. Electoral Studies, 21, 1-22.

Losada, R. (2012). A propósito de la representación política en el nivel local en Colombia : teoría vs. realidad. En I. Cheresky (Ed.), ¿Qué Democracia en América latina? (pp. 191-202). CLACSO; Prometeo libros.

Luján, D. I., \& Schmidt, N. (2018). Volatilidad electoral y alternancia política a nivel subnacional en Uruguay, 2000-2015. Revista Mexicana de Ciencias Politicas y Sociales, 232, 219-246. https://doi.org/10.22201/fcpys.2448492xe.2018.232.58860

Mainwaring, S., Gervasoni, C., \& España-Najera, A. (2017). Extra- and within-system electoral volatility. Party Politics, 23(6), 623-635. https://doi. org/10.1177/1354068815625229 
Mainwaring, S., Gervasoni, C., \& España-Nájera, A. (2010). The vote share of new and young parties (N.o 368). https://kellogg.nd.edu/sites/default/files/old_files/ documents/368_0.pdf

Mainwaring, S., y Zoco, E. (2007). Secuencias políticas y estabilización de la competencia partidista: Volatilidad electoral en viejas y nuevas democracias. América latina hoy, 46(Agosto), 147-171.

Martín de la Cruz, M., y García de Madariaga, J. (2019). ¿Cómo influye el liderazgo político en el comportamiento electoral subestatal? Una aproximación cualitativa. Cuadernos de gobierno y administración pública, 6, 163-184.

Milanese, J. P., y Jaramillo, L. E. (2014). Impacto de los factores institucionales del sistema electoral en la fragmentación partidaria. Un análisis de las elecciones para concejos municipales en el Valle del Cauca (1997-2011). Colombia Internacional, 84(Mayo-Agosto), 43-70. https://doi.org/10.7440/ colombiaint84.2015.02

Montabes, J. (1996). La concurrencia electoral en andalucía. Estudios regionales, 4, 353-370.

Montero, J. (1988). Voto nacional y voto autonómico: la escisión del voto en las elecciones de 1986 en Andalucía. Reis. Revista Española de Investigaciones Sociológicas, 42, 177-194.

Montero, J. R., y Font, J. (1991). El Voto Dual en Cataluña: Lealtad y Transferencia de Votos en las Elecciones Autonómicas. Revista de Estudios Políticos (Nueva Época), 73 (julio - sept), 7-34.

Muñoz, P., Fortou, J., Preciado, A., Ramírez, M., Duque, J., Urreste, J., Milanese, J., Fernández, J., Guzmán, C., Cardona, P., Patiño, L., Silva, M., Vieco, L., Casas, A., Méndez, N., Chasquetti, D., y Murillo, G. (2013). LA CIENCIA POLÍTICA EN COLOMBIA: ¿una disciplina en institucionalización? (S. Leyva (Ed.); Primera). Colciencias, Asociación colombiana de ciencia política, Centro de análisis político EAFIT.

Ocaña, F. A., y Oñate, P. (1999). Índices e indicadores del sistema electoral y del sistema de partidos.Una propuesta informática para su cálculo. Reis. Revista española de investigaciones sociológicas, 86, 223-245.

Pallarés, F., Riba, C., y Fraile, M. (2000). Variables sociestructurales y comportamiento electoral en las elecciones generales españolas. Una perspectiva evolutiva 1979-2000. Revista de Estudios Políticos (Nueva Época), 135(eneromarzo), 109-158.

Reif, K., \& Schmitt, H. (1980). Nine Second-order National Elections - A Conceptual Framework for the Analysis of Euroepan Election Results. European Journal of Political Research, 8(1), 3-44. https://doi. org/10.1111/j.1475-6765.1980.tb00737.x
Resultados electorales municipales 2011-2019. (s. f.). https:// www.registraduria.gov.co/-Historico-de-Resultados,3635-html

Riba, C. (2000). Voto dual y abstención diferencial. Un estudio sobre el comportamiento electoral en Cataluña. Reis. Revista Española de Investigaciones Sociológicas, 91, 59-88.

Riba, C., y Boix, C. (2000). Las Bases Sociales Y Políticas De La Abstención En Las Elecciones Generales Españolas: Recursos Individuales, Movilización Estratégica E Instituciones Electorales. Reis, 90, 95-128. https://doi.org/10.2307/40184253

Riera, P. (2009). Non bis in idem: Voto escindido en sistemas electorales mixtos. Los casos de Nueva Zelanda en 1999 y 2002. Revista Española de Ciencia Política, 20(20), 97-123.

Riera, P., Gómez, R., Barberá, P., Mayoral, J. A., y Montero, J. R. (2016). Elecciones municipales en España: Un análisis multinivel de los determinantes individuales y contextuales del voto. Revista de Estudios Politicos, 172, 47--82. https://doi.org/doi: http://dx.doi.org/10.18042/cepc/rep.172.02

Rodríguez, C. R., y García, D. (2016). Elecciones del 2015 en Colombia: Partidos Políticos y selección de candidatos en el ámbito subnacional. Analisis Politico, 88(septiembre-diciembre), 3-27.

Roscoe, D. (2008). The Choosers or the Choices? Voter Characteristics and the Structure of Electoral Competition as Explanations for Ticket Splitting. The Journal of Politics, 65(4), 1147-1164.

Ruiz, L., y Otero, P. (2013). Indicadores de partidos y sistemas de partidos. Cuadernos Metodológicos CIS, 51 .

Sanz, A. (2007). La escisión del voto en la competición electoral multinivel de la Rioja: elecciones municipales y autonómicas, 1983-2007. Anuario Jurídico de La Rioja, 12, 91-117.

Sanz, A. (2008). La escisión vertical del voto en la competición electoral multinivel: elecciones generales y autonómicas en Andalucía. Revista Española de Ciencia Política, 19, 169-198.

Trujillo, J. M., Ortega, C., y Montabes, J. (2015). Tipo de hábitat y comportamiento electoral: los efectos contextuales sobre la abstención diferencial en Andalucía (2011-2012). Revista española de ciencia política, 37(Marzo), 31-61. 